\title{
Crash course in prescribing - an interactive case-based teaching for final year medical students
}

\author{
Authors: Yueqi Ge, ${ }^{\mathrm{A}}$ Frances Rogerson, ${ }^{\mathrm{A}}$ Chetan Mehta ${ }^{\mathrm{A}}$ and Abdem Rahim Elniel ${ }^{\mathrm{A}}$
}

\section{Introduction}

Prescribing is an essential skill for all newly qualified doctors. With an expanding national formulary and ageing population bringing added pressures of polypharmacy and complex health needs, there are now greater demands to prescribe drugs safely and effectively. Despite being outlined as a core competency in the General Medical Council (GMC)'s Outcomes for Graduates,' literature shows that the majority of medical graduates do not feel confident in prescribing at the onset of their careers, ${ }^{2}$ a trend reflected during data collection for this project. Most medical school curricula cover clinical pharmacology through didactic lecture-based teaching. ${ }^{3}$ However, studies demonstrate that active learning techniques, such as case or team-based learning, improve knowledge retention and facilitate a smoother transition from theory to real-life problems. ${ }^{4,5}$

The aim of this project was to develop an interactive, case-based prescribing teaching resource aimed at final year medical students to help prepare them for common prescribing situations faced in foundation year 1 (FY1).

\section{Methods}

Newly qualified graduates from a London medical school $(n=18)$ were asked via questionnaire about their confidence in prescribing, the medications they found most difficult to prescribe as newly qualified doctors, and their preferred method of teaching.

An interactive small-group teaching session of current final year medical students $(n=11)$ was designed to address the previous responses. Learning outcomes were clearly stated and mapped to the university curriculum to demonstrate relevance. A fun warmup activity was used to encourage student participation from the outset. Teaching focused on four difficult topics identified by the initial questionnaire: intravenous insulin infusions, blood products, end-of-life care and anticoagulation. Students were given ten minutes to tackle each scenario in small groups to simulate the pressure of working on call, followed by a rotational review session with a tutor.

A pre- and post-session questionnaire, inclusive of a single best answer (SBA) test, was used to assess student knowledge, confidence and interactivity of the teaching session. All data were collected using Likert scales via questionnaire.

Authors: ${ }^{A}$ Epsom and St Helier University Hospitals NHS Trust, Epsom, UK

\section{Results and discussion}

Students' responses ( $n=11$; all in brackets on a scale from 1 to 5 , where 1 is least and 5 is most) showed an increase in mean confidence levels in prescribing after completing the session (1.84 to 3.61). They found the session more interactive (4.73) and useful (4.81) than previously encountered prescribing teaching sessions (3.36 and 3.72 respectively). Scores in the SBA test increased from a mean of $46.59 \%$ to $79.55 \%$ after the session. Moreover, they felt that it was more representative of prescribing as an FY1 doctor compared to previous teaching (4.72 compared to 3.36 ).

\section{Conclusion}

The use of interactive case-based learning in prescribing teaching helped to improve student knowledge of targeted areas highlighted by their predecessors. Compared to traditional didactic teaching, the students described increased confidence and felt more prepared for real-life prescribing on wards as newly qualified doctors. By making the session interactive and time-pressured, it simulates the typical hospital environment, and enables students to exercise skills necessary to deliver safe and effective prescribing as an FY1 doctor.

\section{Conflicts of interest}

None declared.

\section{References}

1 General Medical Council. Outcomes for graduates. GMC, 2018. www.gmc-uk.org/-/media/documents/dc11326-outcomes-forgraduates-2018_pdf-75040796.pdf

2 Heaton A, Webb DJ, Maxwell SRJ. Undergraduate preparation for prescribing: the views of 2413 UK medical students and recent graduates. Br J Clin Pharmacol 2008;66:128-34.

3 Maxwell S, Walley T. Teaching safe and effective prescribing in UK medical schools: a core curriculum for tomorrow's doctors. $\mathrm{Br}]$ Clin Pharmacol 2003;55:496-503.

4 Kulak, Newton, Sharma. Does the Use of case-based learning impact the retention of key concepts in undergraduate biochemistry? High Educ 2017;6:110-20.

5 Cevik AA, ElZubeir M, Abu-Zidan FM, Shaban S. Team-based learning improves knowledge and retention in an emergency medicine clerkship. Int J Emerg Med 2019;12:6. 